\title{
Experimental investigation of high speed granular flows down inclines
}

\author{
Joris Heyman ${ }^{1,2}$, Philippe Boltenhagen ${ }^{1}$, Renaud Delannay ${ }^{1}$, and Alexandre Valance ${ }^{1, \star}$ \\ ${ }^{1}$ Institut de Physique de Rennes, CNRS UMR 6251, University of Rennes 1, 35042 Rennes Cedex, France \\ ${ }^{2}$ Géosciences Rennes, CNRS UMR 6118, University of Rennes 1, 35042 Rennes Cedex, France
}

\begin{abstract}
We report on laterally confined granular flow experiments on steep slopes. We provide evidences for the existence of different flow regimes with secondary flows. At moderate mass flow, we observe a first flow regime with a pair of longitudinal vortices which are localized close to the lateral walls and span progressively over the whole flow width with increasing flow rate. They are counter-rotative and induce a vertical upward motion of the grains at the wall. Upon a further increase of the mass flow rate, a transition is evidenced by a reversal of the rotation direction of the vortices which trigger in contrast a downward motion of the grains close to the lateral walls and an upward motion at the center of the channel. We argue that these flows bear some resemblance with the flow regimes reported recently in discrete element simulations.
\end{abstract}

\section{Introduction}

The inclined plane geometry forms particularly interesting boundary conditions for granular flows since, in addition to being comparable to several industrial and natural flows, it may be seen as a rheological test with constant friction. To date, experiments and simulations have focused mainly on mildly sloping planes, leading to fairly well understood flow regimes. More complex flows, including spanwise vortices [1-3], were obtained at slightly higher angles suggesting that upon further steepening, granular flows may reveal original features.

Recent discrete element method simulations of granular flows down steep and flat inclines $[4,5]$ revealed the existence of new flow regimes characterized by complex internal structures: secondary flows, heterogeneous particle volume fraction, symmetry breaking and dynamically maintained order. One of these regimes, referred to as "supported flow", is particularly interesting: it exhibits an uncommonly high bulk velocity, the granular flow being "supported" on a small collisional gaseous layer of highly agitated grains. Similarly to an air-cushion suspension, this layer reduces the effective wall friction and increases significantly the bulk velocity. These "supported" flows have extremely interesting potential applications in geophysics. The reduction in the effective friction due to the gaseous granular layer could indeed explain unexpected long run-out distances of large granular avalanches. However, although this hypothesis was suggested a long time ago by Campbell [6], no experimental evidences of these peculiar flow regimes have been constituted so far.

We present in this article preliminary experimental results on high speed granular flows. We provide evidences for the existence of different types of flow regimes with

\footnotetext{
^e-mail: alexandre.valance@uni-rennes1.fr
}

secondary flows which bear some similarities with the supported regimes recently reported in numerical simulations [5].

\section{Experimental set-up}

The experiments were achieved using a $1.5 \mathrm{~m}$ long granular chute flow facility with lateral glass walls. The inclination $\theta$ of the chute can be varied from a few degrees to $50^{\circ}$ and the spacing $W$ between the lateral walls is also tunable from a few centimeters to a few decimeters. The chute facility is equipped with an automatic particle conveyor allowing to reach high flow discharges in steady configuration. The granular material used in the experiments is spherical glass beads of diameter $d=1 \mathrm{~mm}$ and density $\rho_{p}=2650 \mathrm{~kg} / \mathrm{m}^{3}$.

The presence of lateral walls plays a major role for the observation of steady and fully developed flow regimes on relative short distances even for steep slopes. In fact, the additional lateral friction ensures the existence of stable and stationary flow regimes even for steep slopes as shown in recent simulations [5]. Moreover, the higher the lateral confinement is, the shorter is the transient to reach the steady regime. We thus carried out granular flow experiments on steep slopes with a rather strong confinement (i.e., small wall spacing) but nevertheless not too strong to allow for the apparition of secondary flow regimes. According to numerical simulations [7], a good compromise for the wall spacing is $W=44 d$ where $d$ is the grain diameter.

The base of the chute consists of a flat and smooth aluminum substrate. The flow rate is accurately controlled by a hopper (see Fig. 1) installed at the entrance of the chute and set at $10 \mathrm{~cm}$ above the chute base. The speed of conveyor belt is tuned such that the hopper is always filled 


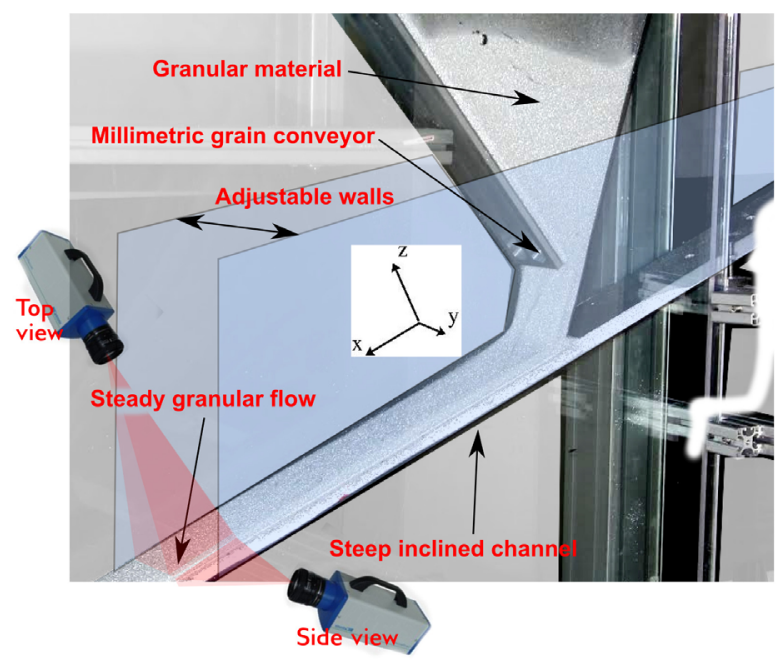

Figure 1. Photography of the granular chute flow set-up: the flow runs over a smooth inclined base and is confined between two lateral walls. The $x$ direction corresponds to the main flow direction, the $y$ direction is perpendicular to the lateral side wall and the $z$ direction is normal to the flow base.

up with grains. This system ensures a steady flow rate for indefinite time.

The granular flow was characterized using a high speed video camera (APX RS photron) located at the end of the chute where the flow is expected to be fully developed. We recorded videos at a frame rate of 10000 frames per second from the transparent side walls but also from above. Using a self-developed particle tracking velocimetry algorithm, we were able to extract particle velocity and particle velocity fluctuations at the lateral wall and at the free surface (i.e., particle-air interface). In addition, we were able to assess the particle concentration at the wall.

\section{Experimental Results}

We report below the features of steady and fully developed granular flows obtained at an inclination angle $\theta=30^{\circ}$. Fig 2 presents the transverse profiles of the two components $u$ and $v$ (along the $x$ and $y$ direction, respectively) of the averaged particle velocity measured at the free surface. The longitudinal velocity $u$ exhibits a parabolic-like profile at moderate flow rate but changes to a plug flow (flat profile) at higher flow rate (see Fig. 2.a).

Finite transverse velocity $v$ informs us of the presence of secondary flows. At moderate flow rate, we observe two symmetrical lobes in the transverse velocity profile close to the lateral walls (see Fig. 2.b). These lobes are the signature of the presence of a pair of vortices. The positive and negative sign of the transverse velocity close to the left-hand side and right-hand side wall, respectively, indicate that the particles are moving away from the walls. The vortices are localized first close to the walls and span progressively over the whole flow width with increasing flow rate. At higher flow rate corresponding to a mass hold-up
$H \approx 8 d^{1}$, there is a sudden reversal of the rotation direction of the vortices. The vortices still appear as a pair. At the highest mass flow rate investigated so far, the transverse velocity exhibits a quasi-linear variation with a zero value at the middle of the channel and a maximum value at five grains diameters from the lateral wall.
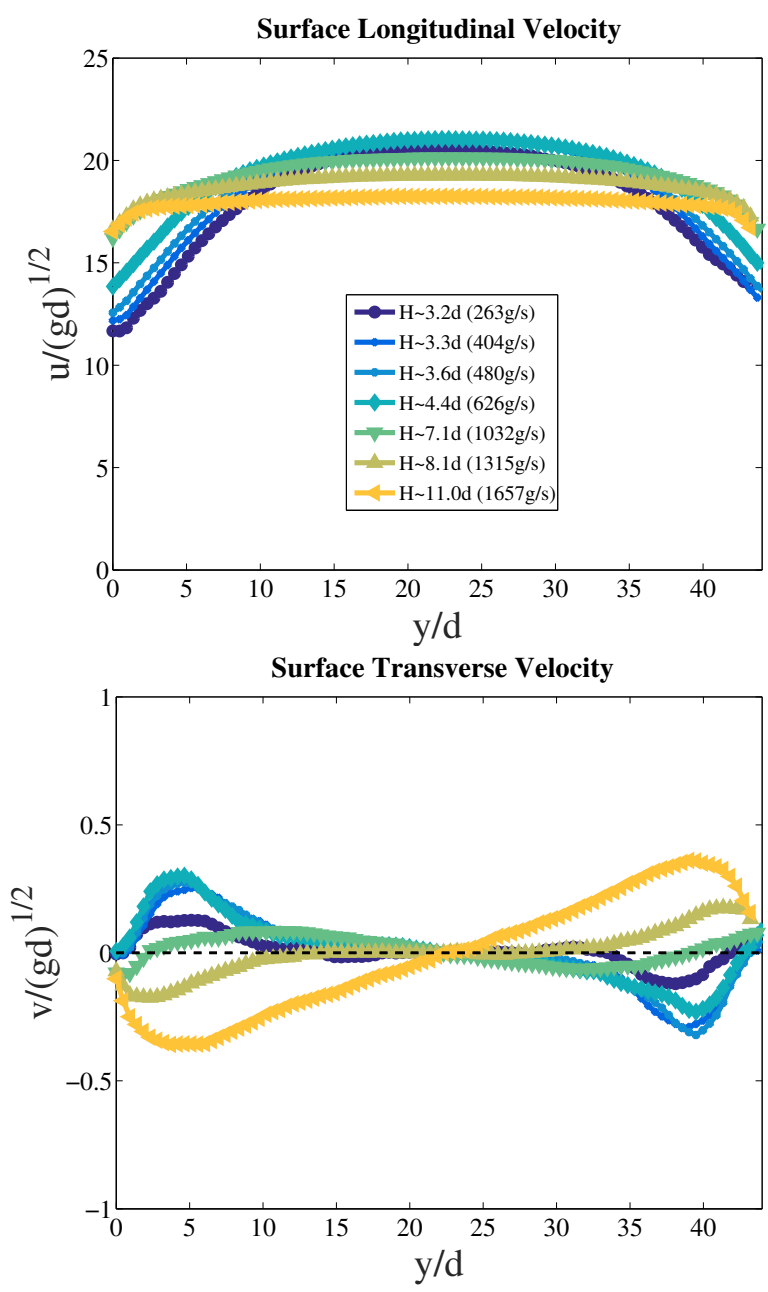

Figure 2. a) Top: transverse profiles of the longitudinal velocity (i.e, $u(y))$ at the free surface for increasing mass flow rate; b) Bottom: transverse profiles of the transverse velocity (i.e., $v(y)$ ) at the free surface for increasing mass flow rate; Chute flow configuration: $W=44 d$ and $\theta=30^{\circ}$.

In Fig 3, we present the corresponding vertical profile of the two components $u$ and $w$ (along the $x$ and $z$ direction, respectively) of the averaged particle velocity measured at the wall. The vertical profile of the longitudinal velocity $u(z)$ indicate a strong slip at the base which decreases with increasing flow rate. At moderate flow rate, the longitudinal velocity exhibits a Bagnold profile (convex curvature), while at higher flow rate, the profile is almost linear with a finite slip velocity (see Fig. 3.a).

The vertical velocity $w(z)$ reveals interesting features which are related with the finite transverse velocity mea-

\footnotetext{
${ }^{1}$ the mass hold-up corresponds to the depth-integrated volume fraction: $H=(1 / W) \int_{0}^{W} d y \int d z \phi(y, z)$, where $\phi(y, z)$ is the particle volume fraction at position $(y, z)$
} 
sured at the free surface. The finite vertical velocity confirms the existence of vortices (see Fig. 3.b). At low flow rate, the positive vertical velocity indicates that the particles are moving upwards and the parabolic shape of the profile informs us that the vortices spread over the whole depth of the flow. In contrast, at higher flow rate, there is a clear reversal of the sign of the vertical velocity in the upper part of the flow, indicating that the particles close to the wall have a downward vertical motion. Furthermore, we clearly see that the vortices observed from the free surface spread only over the upper three quarter of the flow depth. In addition, the $\mathrm{S}$-shape of the velocity profile may suggest the possible emergence of an other vortex with an opposite direction of rotation in the lower quarter of the flow, although the data do not confirm this (since the vertical velocity remains negative). Further experiments are required to confirm this conjecture.
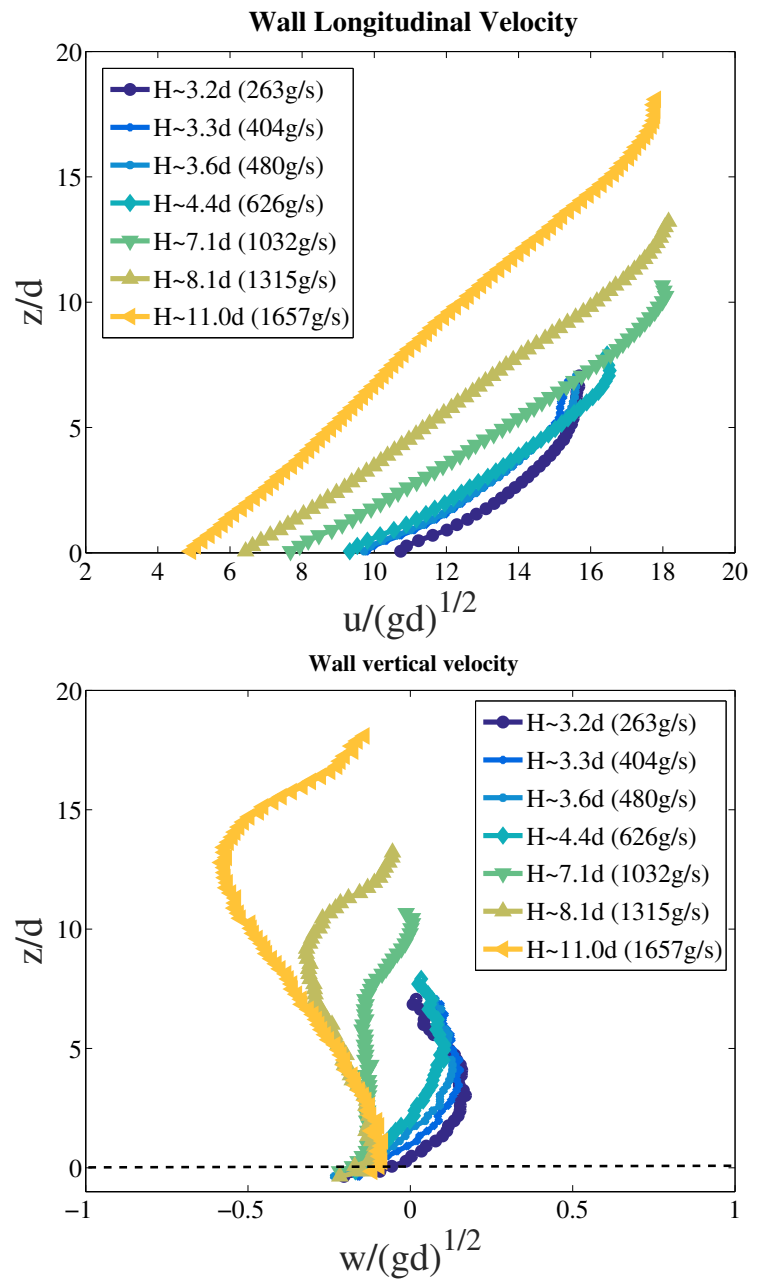

Figure 3. a) Top: vertical profile of the longitudinal velocity (i.e., $u(z)$ ) at the lateral wall; (b) Bottom: vertical profile of the vertical velocity (i.e., $w(z))$ at the lateral wall. Chute flow configuration: $W=44 d$ and $\theta=30^{\circ}$.

It is worthwhile to note that the existence of two superposed rows of vortices is seen in numerical simulations for high mass hold-up[5]. The apparition of an additional pair of vortices is actually characterized by a reversal of the rotation direction of the upper vortices in comparison with the lower ones.

Although, we do see the interior of the flow, the combination of velocity profiles measured at the free surface and at the lateral walls provides us with a rather good description of the secondary flow structure. The structure of the two different flow regimes identified from the velocity measurements is illustrated in Fig. 4. At low flow rate, we have two longitudinal vortices which induce an upward particle motion at the walls and a shift of the particles towards the center of the flow at the free surface. At higher flow rate, we observe a reversal of the rotation direction of the vortex. In the latter case, the vortices do not spread over the whole depth of the flow which may suggest the presence of an other pair of vortices. (b)

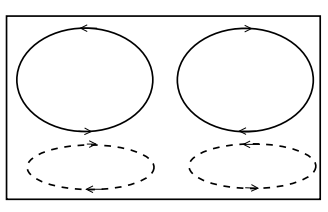

Figure 4. Sketch of the structure of the flow regimes obtained in the experiments: (a) Low flow rate regimes with longitudinal vortices inducing an upward motion of the particles close to the walls $(3<H<8)$; (b) High flow rate regimes with vortices spinning in the reverse direction $(8<H<12)$. The presence of an additional pair of vortices is a conjecture to be checked experimentally.

The last feature extracted from the video recording is the vertical profile of the particle concentration at the wall (see Fig. 5). The 2D image analysis does not provide us with a true measurement of the particle volume fraction but give us a proxy. Stereo imaging would be required to assess accurately the volume fraction. At moderate flow rate, the concentration exhibits a parabolic shape where the bulk flow is denser than the lower and upper layer. The density of the bulk increases with increasing flow rate. At higher flow rate, the concentration is much more uniform within the bulk (flat profile). The other salient feature is the small decrease of the bulk concentration when the mass hold-up is increased from $H=7.1 d$ to $H=8.1 d$. This is a signature of a small depletion of particles close to the walls but we can not rule out the possibility of an artefact of the tracking algorithm.

These preliminary measurements on high speed granular flows do not provide us with a full description of their structure since we can not probe the interior of the flow. On the basis of this partial description, we can not claim with certainty that the observed regimes are similar to the supported flows recently reported in discrete numerical simulations [5]. The latter indicate that the supported flows appear for inclination angle equal to or larger than $30^{\circ}$ and are characterized by a highly concentrated core surrounded by an extremely dilute layer of highly agitated particles [5]. This highly depleted layer is located at the lateral walls and at the flow base and acts similar to a air- 


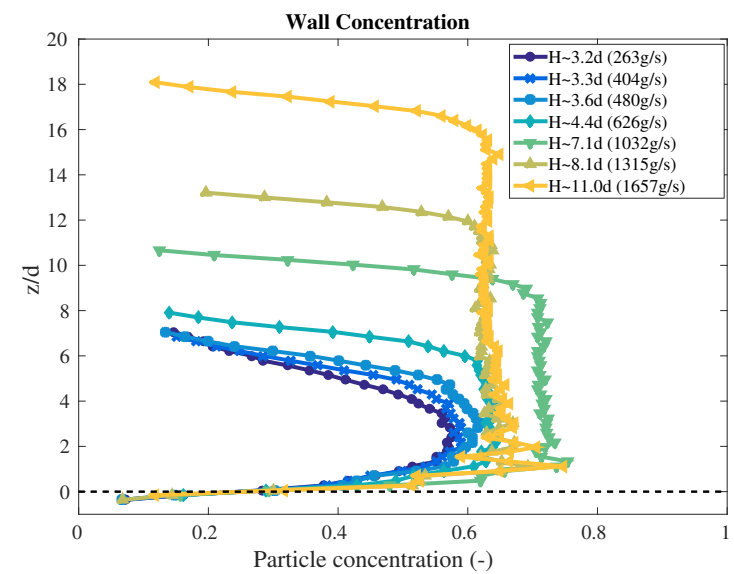

Figure 5. Vertical profile of the particle concentration at the lateral wall. Chute flow configuration: $W=44 d$ and $\theta=30^{\circ}$.

cushion suspension reducing the friction at the walls. At the moment, we do not have clear experimental evidences of the existence of a highly depleted layer at the later wall and at the base. We may suspect that the transition towards the supported flow regime is shifted to higher values of inclination angle when the lateral confinement is increased (i.e., for smaller wall spacing $W$ ) [7]. Actually the simulations of Brodu et al. [5] were performed for a flow width $W=68 d$ whereas the experiments were conducted with a wall spacing $W=44 d$.

\section{Conclusion}

We reported preliminary measurements on granular flow regimes on a steep slope which reveal complex structures with secondary flows as observed in recent numerical simulations [5]. Additional measurements are required to better document these high flow regimes. We plan in the near future to investigate these flows within a broader range of inclination angle and mass flow rate with the aim to provide clear evidences of the existence of the supported flow regime.

Besides, an effort should be made in the development of new experimental techniques to probe the interior of the flow because the classical imaging techniques are limited to the characterization of what happens at the wall and the free surface.

\section{Acknowledgments}

We acknowledge the support of the French "Institut des Sciences de l'Univers" (INSU) through the national research program "Aléas et Risques".

\section{References}

[1] Y. Forterre and O. Pouliquen, Phys. Rev. Lett. 86, 5886 (2001).

[2] Y. Forterre and O. Pouliquen, J. Fluid Mech. 467, 361-387 (2002).

[3] T. Börzsönyi, R.E. Ecke and J.N. McElwaine, Phys. Rev. Lett. 103, 178302 (2009).

[4] N. Brodu, P. Richard and R. Delannay, Phys. Rev. E 87, 022202 (2013)

[5] N. Brodu, R. Delannay, A. Valance and P. Richard, J. Fluid Mech. 769, 218-228 (2015).

[6] C.S. Campbell, J. Geol. 97 , 653-665 (1989).

[7] J.-L. Ralaiarisoa., N. Brodu, R. Delannay and A. Valance, in preparation (2016). 